\title{
ON APPROXIMATE CORES OF NON-CONVEX ECONOMIES
}

\section{Birgit GRODAL}

University of Copenhagen, 1168 Copenhagen $K$, Denmark

\section{Walter TROCKEL}

University of Bonn, 5300 Bonn 1, West Germany

\author{
Shlomo WEBER \\ University of Haifa, Haifa 31999, Israel
}

Received 13 February 1984

In this note we investigate, for non-convex finite economies, the relationship between the existence of approximate core allocations and the size of an economy.

\section{Introduction}

The existence of approximate equilibria for exchange economies whose agents have non-convex preferences was established by Starr (1969). For a stronger concept of approximate equilibria the existence result was obtained by Hildenbrand, Schmeidler and Zamir (1973) (HSZ hereafter). Moreover, it was shown in HSZ, that the approximation can be made as good as one likes by choosing the number of agents in the economy large enough.

Under assumption of completeness of preferences, the analogous existence result for approximate cores was also obtained in HSZ. In a consequent paper Grodal (1976) has extended this result to the non-complete case.

In a recent contribution Anderson (1982) proved a 'rate-of-convergence' theorem for approximate equilibria in the framework of quite general sequences of finite exchange economies, including those se- 
quences considered in HSZ. A special case of Anderson's result was proved subsequently by Weber (1980) for the sequences of HSZ by modifying arguments given already there.

In this note we establish a relationship between the existence of approximate core allocations and the size of a non-convex economy. The result is directly related to the previous papers by HSZ and Grodal (1976).

To obtain our proposition the only additional assumption we need is a cone-monotonicity of preference relations which is defined below.

\section{Model}

We follow HSZ very closely, and all notations, assumptions and definitions, except of $C$-monotonicity, are reproduced from there.

Let $T$ denote an infinite set of potential traders. For every $t \in T$ there are defined:

(i) a preference relation $\succ_{\text {, }}$ on the positive orthant $R_{+}^{l}$ of the $l$-dimensional Euclidean space $R^{\prime}(l \geqslant 2)$,

(ii) a vector of the initial endowment $w_{t}$ in $R_{+}^{\prime} \backslash\{0\}$.

We assume that:

A.1. For all $t \in T, \succ$, is irreflexive, transitive, open [the set $\{(x, y) \in$ $\left.\mathrm{R}_{+}^{1} \times \mathrm{R}_{+} \mid \mathrm{x} \succ_{\mathrm{t}} \mathrm{y}\right\}$ is open in the relative topology of $R_{+}^{I} \times R_{+}^{\prime}$, and strongly monotone $\left(x \in R_{+}^{\prime}, u \in R_{+}^{\prime} \backslash\{0\}\right.$ implies $\left.x+u \succ, x\right)$.

A.2. Uniform boundedness of initial endowments. There is a positive real number $M$ such that $w_{t} \leqslant M e$ for all $t$ in $T$, where $e$ denotes the unit vector $(1, \ldots, 1) \in R^{l}$.

A.3. Compactness of preferences. The set $\left\{\succ_{t}\right)_{t \in T}$ is compact. [For a precise definition of the topology on the set of preferences see HSZ (p. 1164). It is shown there, for example, that the set of all irreflexive, transitive, open, and monotone preferences is compact with respect to this topology.]

Now, let $C \subset R^{\prime}$ be a proper cone such that int $C \supset R_{+}^{\prime} \backslash\{0\}$. [Recall that $C$ is a proper cone if (i) $C+C \subset C$, (ii) $\lambda C C C$ for all $\lambda>0$, (iii) $C \cap(-C)=\{0\}$. Cf. Shaefer (1966).] 
Definition 1. A preference relation on $R_{+}^{\prime}$ is $C$-monotone at $x \in R^{\prime}$, if $\left[u \in C \backslash\{0\}, x+u \in R_{+}^{\prime}\right]$ implies $[x+u>x]$. A preference relation is $C$-monotone on a set $A \subset R_{+}^{\prime}$ if it is $C$-monotone at $x$ for every $x \in A$. A preference relation is $C$-monotone if it is $C$-monotone on $R_{+}^{l}$.

Now denote for any $\lambda \in(0,1)$ and $i \in\{1,2, \ldots, l\}$ the vector<smiles>[Y]C(C)CC(C)CC(C)CC</smiles>

by $e_{\lambda}^{i}$. Then $C_{\lambda}$ denotes the convex cone generated by the set $\left\{0, e_{\lambda}^{1}\right.$, $\left.e_{\lambda}^{2}, \ldots, e_{\lambda}^{l}\right\}$. Note that there is $\lambda \in(0,1)$ such that $C \supset C_{\lambda}$.

We denote by $S$ the open simplex

$$
\left\{p=\left(p^{1}, \ldots, p^{i}\right) \in R_{+}^{l} \mid \sum_{i=1}^{l} p^{i}=1 \text { and } p^{i}>0 \text { for all } i\right\}
$$

in $R_{+}^{\prime}$. The demand correspondence $\psi: T \times S \rightarrow R_{+}^{\prime}$ is defined by

$\psi(t, p)=\left\{x \in \Omega \mid p x \leqslant p w_{t}\right.$ and $y \succ_{t} x$ implies $\left.p y>p w_{i}\right\}$.

It is shown in Schmeidler (1969) that $\psi$ is well defined and, by the monotonicity assumption, $p x=p w_{t}$ holds for all $x$ in $\psi(t, p)$. An exchange economy is by definition a finite subset, say $E$, of $T$. An allocation for the economy $E$ is a collection $\left\{x_{t}\right\}_{t \in E}$ of elements of $R_{+}^{l}$ satisfying $\sum_{t \in E}\left(x_{t}-w_{t}\right)=0$.

In order to state our proposition we need the following:

Definition 2. Let $\epsilon>0$ and an economy $E$ be given. Then the $\epsilon$-core of $E$ is defined to be the set of all allocations $\left\{x_{t}\right\}_{t \in E}$, such that there do not exist a non-empty subset $S$ of $E$ and an allocation $\left\{y_{t}\right\}_{t \in E}$ for $E$ satisfying

(i) $y_{t} \succ_{t} x_{t}$ for every $t \in S$,

(ii) $\sum_{t \in S} y_{t} \leqslant\left(\sum_{t \in S} w_{t}\right) \theta|S| \epsilon e$,

( $|A|$ denotes the cardinality of a set $A$, and for any $x, y \in R_{+}^{i}$ the vector whose $j$ th coordinate is $\max \left\{0, x^{j}-y^{j}\right\}$ for all $1 \leqslant j \leqslant l$ is denoted $x \theta y$.)

Proposition. Let A.1-A.3 be satisfied and $\delta>0$. Suppose that for any $n \in N$ there is $a \lambda_{n}>0$ so that every $\succ_{t}$ is $C_{\lambda_{n}}{ }^{-m o n o t o n e}$ on $[0, n]^{l}$. Then for 
any sequence of economies in $T$ with $\sum_{t \in E_{n}} w_{t}>\left|E_{n}\right|$ Se for each $n$, there are sequences $\left\{\left\{x_{i}^{n}\right\}_{i \in E_{n}}\right\}_{n \in N}$ of allocations and $\left\{\epsilon_{n}\right\}_{n \in N}$ of positive numbers with $\epsilon_{n}=O\left(1 /\left|E_{n}\right|\right)$, such that $\left\{x_{t}^{n}\right\}_{\iota \in E_{n}} \in \epsilon_{n}$-core of $E_{n}$ for every $n \in N$.

\section{Preliminary statements and proof of the proposition}

Let us state the following lemma, which is useful for the proof of the proposition:

Lemma. Let $\delta>0$. Under assumptions A.1-A.3, for any sequence $\left\{E_{n}\right\}_{n \in N}$ of economies in $T$ with $\sum_{t \in E_{n}} w_{t}>\left|E_{n}\right| \delta e$ for each $n$, there are sequences $\left(\left\{x_{l}^{n}\right\}_{t \in E_{n}}, p^{n}\right)_{n \in N}$ of allocations and of prices in $S$, such that for each $n \in N$ and each $t \in E_{n}$ one has $p^{n} x_{t}^{n}=p^{n} w_{t}$ and $\rho\left(x_{t}^{n}, \psi\left(t, p^{n}\right)\right)=$ $0\left(1 /\left|E_{n}\right|\right)$. (For $x \in R^{\prime}$ and $A \subset R^{\prime}, \rho(x, A)$ denotes $\left.\inf _{y \in A}\|x-y\|\right)$.

This Lemma due to Weber (1980) is a corollary of Theorem 2 in Anderson (1982).

Consider the following two statements concerning an irreflexive preference relation $>$ :

(i) for every $x, y, x^{\prime}, y^{\prime} \in R_{+}^{\prime}: y \geqslant x, y_{+}^{+} x,\left\|y-y^{\prime}\right\| \leqslant\|y-x\| / K$ and $\| x$ $-x^{\prime}\|\leqslant\| y-x \| / K \Rightarrow y^{\prime} \succ x^{\prime}$.

(ii) $\succ$ is $C_{\lambda}$-monotone.

Then we can state:

Claim 1. (i) implies that (ii) holds for every $\lambda \leqslant 1 /(l-1)(\sqrt{l} K+1)$.

Claim 2. (ii) implies that (i) holds for $K \geqslant 4 \sqrt{l} / \lambda$.

Proof of Claim 1. Assume (i) is satisfied and consider $x \in R_{+}^{l}, u \in C_{\lambda} \backslash$ $\{0\}$, where $\lambda \leqslant 1 /(l-1)(\sqrt{l} K+1)$. Then, $u=\sum_{i=1}^{l} \delta_{i} e_{\lambda}^{i}$ for some $\delta_{i} \geqslant 0$. Let $\delta=\max _{i=1, \ldots,} \delta_{i}>0$. Now define $y^{\prime}=x+u$ and assume that $y^{\prime} \in R_{+}^{\prime}$. We have to prove that $y^{\prime} \succ x$. Therefore define $v \in R_{+}^{l}$ by $v_{i}=\max \left(0, \delta_{i}\right.$ $\left.-\lambda \sum_{h \neq i} \delta_{h}\right)$ for $i=1, \ldots, l$ and let $y=v+x$. Then, $\|y-x\|=\|v\| \geqslant \delta$ $-\lambda(l-1) \delta$. Moreover $\left\|y^{\prime}-y\right\|=\|u-v\| \leqslant\left\|\left(\sum_{h \neq i} \lambda \delta_{h}\right)_{i=1}^{\prime}\right\| \leqslant\left((l-1)^{2} \delta^{2} \lambda^{2} l\right)^{\frac{1}{2}}=(l-1) \delta \lambda \sqrt{l}$. 
Since $\lambda \leqslant 1 /(l-1)(K \sqrt{l}+1)$, we have that

$(l-1) \delta \lambda \sqrt{l} \leqslant \frac{\delta-\lambda(l-1) \delta}{K} \leqslant \frac{\|y-x\|}{K}$.

Consequently, as $y \geqslant x$, we have by (i) that $x+u>x$. Q.E.D.

Proof of Claim 2. Assume (ii) and consider $x, y, x^{\prime}, y^{\prime} \in R_{+}^{l}$ such that $y \neq x, y>x$, and $\max \left(\left\|y-y^{\prime}\right\|,\left\|x-x^{\prime}\right\|\right) \leqslant\|y-x\| / K$, where $K \geqslant$ $4 \sqrt{l} / \lambda$. It suffices to show that $\underline{y}-\bar{x}^{\prime} \in C_{\lambda}$, where $\underline{y}^{\prime}:=y(-\|y-x\|$ $/ K)(1, \ldots, 1), \bar{x}^{\prime}:=x+(\|y-x\| / K)(1,1, \ldots, 1)$ (clearly $y^{\prime} \geqslant y^{\prime}$ and $\bar{x}^{\prime}$ $\left.>x^{\prime}\right)$. Note that $y \geqslant x$ implies that $y_{h} \geqslant x_{h}$ for all $1 \leqslant h \leqslant l$, and there exists $i, 1 \leqslant i \leqslant l$, such that $y_{1} \geqslant x_{i}+\|y-x\| / \sqrt{l}$. Therefore, we have that $y^{\prime} \geqslant \bar{x}^{\prime}+(\|y-x\| / \sqrt{l})(0,0, \ldots, 1,0, \ldots, 0)-2(\|y-x\|$ $/ K)(1, \ldots, 1)$. Let

$u=\frac{\|y-x\|}{2 \sqrt{l}}\left(\frac{-4 \sqrt{l}}{K}, \ldots, \frac{-4 \sqrt{l}}{K}, 2\left(1-\frac{2 \sqrt{l}}{K}\right), \frac{-4 \sqrt{l}}{K}, \ldots, \frac{-4 \sqrt{l}}{K}\right)$.

Consider $v \in R^{l}$, where

$v=\left(\frac{-4 \sqrt{l}}{K}, \ldots, \frac{-4 \sqrt{l}}{K}, 1, \frac{-4 \sqrt{l}}{K}, \ldots, \frac{-4 \sqrt{l}}{K}\right)$.

Since $4 \sqrt{l} / K<\lambda<1$, we conclude that $v \in C_{\lambda} \backslash\{0\}$. Consequently, since $\underline{y}^{\prime} \geqslant \bar{x}^{\prime}+u \geqslant \bar{x}^{\prime}+(\|y-x\| / 2 \sqrt{l}) v$, it follows that $\underline{y}^{\prime}-\bar{x}^{\prime} \in C_{\lambda}$. As, moreover, $y^{\prime} \in R_{+}^{\prime}$ and $y^{\prime} \neq x^{\prime}$, we obtain that $y^{\prime}>x^{\prime}$. ${ }^{\prime}$ Q.E.D.

Proof of the proposition. Let $\delta>0$ and let a sequence of economies $\left\{E_{n}\right\}_{n \in N}$ with $\sum_{t \in E_{n}} w_{t}>\left|E_{n}\right| \delta e$ be given. Then, by the lemma, there exist an independent constant $H$ and sequences $\left\{\left\{x_{t}^{n}\right\}_{t \in E_{n}}, p^{n}\right\}_{n \in N}$ of allocations and prices, so that for each $n \in N$ and each $t \in E_{n}$ one has $p^{n} x_{t}^{n}=p^{n} w_{t}$ and $\rho\left(x_{t}^{n}, \psi\left(t, p^{n}\right)\right) \leqslant \alpha_{n}$, where $\alpha_{n}=H /\left|E_{n}\right|$. Let $\eta=\eta(\delta / 2)$ be determined by Lemma 2 of HSZ. Denote by $Q$ a minimal integer, which exceeds $M / 2$. Let $B$ be a cube $[0, Q]^{l}$ and $\lambda_{Q}>0$, so that each preference relation $\succ_{t}, t \in T$, is a $C_{\lambda_{e}}$-monotone on $B$.

Consider an economy $E_{n}$, which belongs to the above sequence of economies. Suppose $x, y, x^{\prime}, y^{\prime} \in R_{+}^{l}, y \geqslant x,\|y-x\|=\left(4 \sqrt{l} / \lambda_{Q}\right) \alpha_{n}$. Then by Claim $2, \max \left(\left\|x-x^{\prime}\right\|,\left\|y-y^{\prime}\right\|\right) \leqslant \alpha_{n}$ implies that $y^{\prime} \succ_{t} x^{\prime}$ for any $t \in E_{n}$. Denote $\beta_{n}=\left(4 \sqrt{ } l / \lambda_{Q}\right) \alpha_{n}$ and $\epsilon_{n}=2 \beta_{n} / \eta(\delta / 2)=$ $(2 / \eta(\delta / 2))\left(4 \sqrt{l} / \lambda_{Q}\right)\left(H /\left|E_{n}\right|\right)$ 
Thus, applying the standard arguments used in Grodal (1976), we conclude that an economy $E_{n}$ has a non-empty $\epsilon_{n}$-core, and this completes the proof of proposition. Q.E.D.

Remark. Let us note, that although for any $\lambda>0$ every $C_{\lambda}$-monotone preference relation is strongly monotone, but for given $\bar{\lambda}>0$, a strongly monotone preference relation cannot, in general, be approximated by a sequence of $C_{\bar{\lambda}}$-monotone preference relations. This can be illustrated by the following:

Example. Let $\bar{\lambda}>0$. Define $>$ on $R_{+}^{\prime}$ by $x>y \Leftrightarrow x-y \in \operatorname{int} C_{\bar{\lambda} / 2}$. (Clearly, $\succ$ is strongly monotone.) Consider a sequence $\left(\succ_{n}\right)_{n \in N}$ of $C_{\bar{\lambda}}$-monotone preference relations and for each $n \in N$ denote $G r\left(>_{n}\right):=$ $\left\{(x, y) \mid x \succ_{n} y, x \in R_{+}^{\prime}, y \in R_{+}^{l}\right\}$. Then $\left[x, y \in R_{+}^{l}, x-y \in C_{\bar{\lambda}}, x \neq y\right]$ implies that $\left[x \succ_{n} y, \forall n \in N\right]$. Moreover, $G r\left(\succ_{n}\right) \supset\left\{(x, y) \mid x-y \in C_{\bar{\lambda}}\right.$ $\left.\backslash\{0\}, y \in R_{+}^{\prime}, x \in R_{+}^{\prime}\right\}$ for all $n$ and since $R_{+}^{\prime} \times R_{+}^{\prime} \backslash \operatorname{Gr}\left(\succ_{n}\right)$ is closed we have that $\left(\succ_{n}\right)$ does not converge to $\succ$.

\section{References}

Anderson, Robert M., 1982, A market value approach to approximate equilibria, Econometrica 50, 127-135.

Grodal, Birgit, 1976, Existence of approximate cores with incomplete preferences, Econometrica 44, 829-830.

Hildenbrand, Werner, David Schmeidler and Shmuel Zamir, 1973, Existence of approximate equilibria and cores, Econometrica 41, 1159-1166.

Schaefer, Helmut H., 1966, Topological vector spaces (MacMillan, New York).

Schmeidler, David, 1969, Competitive equilibria in markets with a continuum of traders and incomplete preferences, Econometrica 37, 578-585.

Starr, Ross M., 1969, Quasi-equilibria in markets with non-convex preferences, Econometrica 37, 25-38.

Weber, Shlomo, 1980, A short note on approximate equilibria and approximate cores, Discussion paper no. 65, SFB 21 Projektgruppe 'Theoretische Modelle' (University of Bonn, Bonn). 